\title{
Microscopio como Lector de Absorbancia con Utilidad en Análisis Clínicos
}

\author{
Microscope as Absorbance Reader with Utility in Clinical Analysis
}

\author{
I. J. Orlando-Guerrero', C. H. Bravo-Delgado', Z. J. Hernández-Paxtián', A. A Aguilar-Felipe² \\ Universidad de la Cañada \\ ¿2aboratorio Clínico Huatulco
}

\section{RESUMEN}

La descentralización de laboratorios clínicos, se encuentra en desarrollo, lo anterior ha llevado a diseñar instrumentos que ofrecen resultados rápidos, confiables y al lado del paciente, esta tendencia se le conoce como prueba en el punto de atención (point of core testing POCT) y brinda la posibilidad de dar un seguimiento inmediato al padecimiento. El objetivo de esta investigación fue la implementación de un medidor de absorbancia, empleando la estructura de un microscopio óptico, al cual se le ha adaptado un filtro de luz, y una cámara digital. Lo anterior permite obtener valores de intensidad promedio de imágenes sólidas microscópicas de reacciones enzimáticas, y a partir de ellas estimar absorbancia o concentración. Como resultados se presentan rectas de calibración de absorbancia y mediciones de concentraciones para los casos de violeta de genciana, un kit de glucosa oxidasa y muestras problemas de pacientes voluntarios. Concluimos que existe un error de medición menor de $\pm 1 \mathrm{mg} / \mathrm{dL}$ comparados con las mediciones de un lector de placas de Elisa y un analizador de química sanguínea semiautomatizado. Teniendo en cuenta lo anterior el sistema resulta ser una alternativa viable para la estimación de absorbancia y aumenta la funcionalidad de microscopios ópticos en clínicas de salud.

PALAbras Clave: Prueba en el punto de atención; lector de absorbancia; micro-espectrofotómetria 


\section{ABSTRACT}

The decentralization of clinical laboratories is under development, which has led to the design of instruments that offer fast, reliable and patient-side results, this trend is known as point of core testing (POCT) and It offers the possibility of giving an immediate follow-up to the disease. The objective of this investigation was the implementation of an absorbance meter, using the structure of an optical microscope, to which a light filter and a digital camera have been adapted. This allows to obtain values of average intensity of solid images of enzymatic reactions, and from them to estimate absorbance or concentration. As results, we present absorbance calibration lines and concentration measurements for cases of gentian violet, a glucose oxidase kit and samples of volunteer patients. We conclude that there is a measurement error of less than $\pm 1 \mathrm{mg} / \mathrm{dl}$ compared with the measurements of an Elisa plate reader and a semi-automated blood chemistry analyzer. Taking into account the above, the system turns out to be a viable alternative for estimating absorbance and increasing the functionality of optical microscopes in health clinics.

KEYwORDS: Point of Core Testing: Absorbance Reader; Microspectrophotometer

\section{Correspondencia}

DESTINATARIO: Israel Jesús Orlando Guerrero

INSTITUCIÓN: Universidad de la Cañada

DIRECCIÓN: Carretera Teotitlán-San Antonio

Nanahuatipán Km 1.7 S/N, Paraje Titlacuatitla, C.P.

68540, Teotitlán de Flores Magón, Oaxaca, México

CORREOELECTRÓNICO: iorlando@unca.edu.mx

\section{Fecha de recepción:}

28 de agosto de 2018

Fecha de aceptación:

11 de enero de 2019 


\section{INTRODUCCIÓN}

El empleo de microscopios invertidos se ha difundido ampliamente en los últimos años, esto debido a que se pueden observar cultivos celulares sin una previa preparación, dicha actividad puede ser resaltada gracias a técnicas de contraste de fase, con las cuales se evita el uso de biomarcadores. Sin embargo, los microscopios ópticos no solo pueden ser empleados para observación, también permiten cuantificar concentraciones de solutos presentes en una célula, tal es el caso de la reciente técnica llamada micro-espectrofotómetria UV-Vis ${ }^{[1]}$, esta técnica añade un valor sustancial a microscopios ópticos, ya que no lo limita a la observación, sino que también expande sus capacidades como un medidor de absorbancias o concentraciones. Lo anterior permite a clínicas de salud tener una doble funcionalidad en un solo instrumento y por ende realizar análisis rápidos, confiables y al lado del paciente, como lo dicta la tendencia POCT ${ }^{[2]}$.

En la presente investigación se emplea un microscopio invertido como un lector de absorbancia, esto se logra añadiendo al sistema un filtro de luz, una cámara digital CMOS para la captura de imágenes digitales sólidas y un análisis numérico de las mismas.

\section{Sensor CMOS como medidor de absorbancia}

La absorbancia (A) es función del registro de intensidades que son obtenidas por el detector, es decir, es una comparación entre la intensidad de referencia (Ir) (intensidad medida en el detector sin muestra) y la intensidad de la muestra (I). La absorbancia puede ser calculada en función de dichos términos, aplicando la conocida ley de Lambert Beer como sigue ${ }^{[3]}$ :

$$
A=\log _{10} I_{r} / I
$$

Por ser una comparación, la sensibilidad del detector es importante en los límites inferiores y superiores de medición admisibles del detector, por ejemplo, si el detector es capaz de medir absorbancias del orden de 5 , significa que este puede medir intensidades muy bajas del orden de $0.001 \%$ de la intensidad de referencia, lo anterior permite medir concentraciones muy altas de un soluto. Por el contrario, absorbancias del orden de 0.001 significa que el detector debe ser capaz de medir intensidades altas del orden de $99.77 \%$ de la intensidad de referencia, por lo tanto, puede medir concentraciones muy bajas de un soluto ${ }^{[3]}$.

Habitualmente en espectrofotometría se emplean fotomultiplicadores como detectores, los cuales generan una corriente que es proporcional a la intensidad incidente, por lo tanto, el cálculo de la absorbancia se realiza empleando la Ecuación (1), pero en términos de intensidad de corriente.

Sin embargo, en la actualidad las cámaras digitales CMOS han empezado a remplazar este tipo de detectores ya que pueden ser empleadas como discriminadores de niveles de luz, es decir como sensores de luminancia. Para este caso, la luminancia es calculada a partir de la siguiente ecuación:

$$
L=f^{2} N / k t s
$$

Dónde: L es la luminancia en candela/metro ${ }^{[2]}, \mathrm{N}$ es el valor del pixel en la imagen digital en formato 8 o 16 bits, $t$ es la velocidad de obturación (cuadros por segundos), f es el número de apertura, $\mathrm{S}$ es la sensibilidad del detector ISO, y k es una constante de calibración obtenida a partir de luminancias estándares ${ }^{[4]}$.

Debido a que la absorbancia es obtenida por una división, esta resulta ser la misma en términos de luminancia, valor de pixel en 8 bits o 16 bits. Lo anterior se confirma en la Tabla 1, donde se muestra un valor de I e Ir y su cálculo de absorbancia en términos de las tres magnitudes. Debido a que es posible estimar la absorbancia en niveles de gris, en esta investigación usamos el formato de valor de pixel de 8 bits para su cálculo. 
TABLA 1. Calculo de absorbancia, empleado la luminancia y dos formatos del valor del pixel.

\begin{tabular}{|cccc|}
\hline & Pixel 8 & Pixel 16 & Luminancia \\
\hline $\mathbf{I}$ & 235 & 60395 & 148208.589 \\
\hline $\mathbf{I}_{\mathbf{r}}$ & 255 & 65635 & 160822.0859 \\
\hline $\mathbf{A}$ & 0.036 & 0.036 & 0.036 \\
\hline
\end{tabular}

Por otro lado, el rango de medición inferior de absorbancia de una CMOS, puede estar limitado por la corriente de oscuridad, la cual es debida a electrones generados térmicamente en total oscuridad, por ejemplo, la CMOS empleada en esta investigación, tiene un ruido promedio de oscuridad de $2 \pm 1$ pixel en niveles de gris, lo cual equivale a una medición de absorbancia de 1.4065, considerando una intensidad de referencia de 255. El límite superior de medición de absorbancia, está limitado por la diferenciación entre niveles de gris a intensidades altas, para este experimento se observaron diferencia significativa entre niveles de gris hasta $251 \pm 1$ pixel, lo cual equivale a una absorbancia de 0.0068 . Por lo anterior el rango de medición de absorbancia de la CMOS es de 2.0986 a 0.0068 .

\section{METODOLOGÍA}

\section{Sistema óptico}

Para la generación de las imágenes sólidas de solutos, se empleó un microscopio invertido marca Olympus modelo CKX 41 ${ }^{[5]}$, (ver Figura 1a), trabajando en campo claro, la descripción del funcionamiento del MLA (microscopio medidor de absorbancia) se describe a continuación: La luz incoherente proveniente de la fuente de tungsteno es filtrada a luz cuasi cromática por medio de un filtro pasa banda modelo FB51010-Ø1 (marca thorlabs ${ }^{[6]}$ ), cuya longitud de onda central es de $\mathrm{CWL}=510 \pm 2 \mathrm{~nm}$, y un ancho medio de $\mathrm{FWHM}=10 \pm 2 \mathrm{~nm}$, este filtro es insertado en el control deslizante modelo IX2-SLP en la poción reservada para iluminación en campo claro, las otras dos posicio- nes empleadas para iluminación en contraste de fase no son empleadas en el experimento, lo anterior se muestra en la Figura $1 b$.

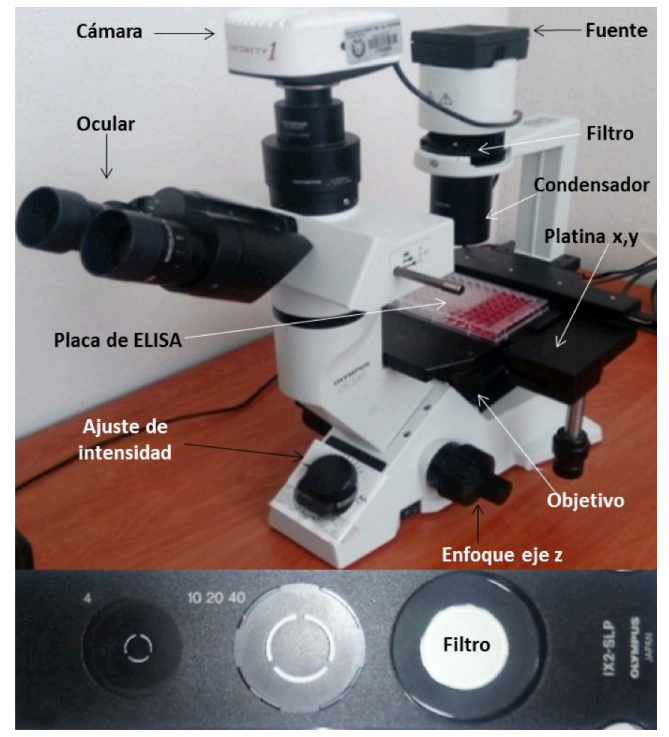

a)

b)

FIGURA 1. a) Sistema optico empleado para la captura de imágenes solidas de reacciones enzimaticas y b) filtro y control dezlizante para iluminacion cuasi cromática.

Posteriormente la luz cuasi cromática es dirigida a una placa de ELISA de 96 posos por medio de un condensador que genera iluminación tipo Köhler, cada poso puede ser seleccionado por el usuario por la platina $x, y$ del microscopio. La intensidad e imagen de cada poso es colectada por medio del objetivo de $60 \mathrm{x}$ (N.A=0.85), de la marca edmundoptics y dirigida a una cámara digital CMOS Infinity 1 de 2.0 megapixeles de la marca lumera, con tamaño de pixel de $4.2 \mu \mathrm{mx} 4.2 \mu \mathrm{m}$ y una resolución de imagen de $1600 \times 1200$ pixeles ${ }^{[7]}$. La intensidad promedio y la absorbancia para cada poso de la imagen digital es calculada por medio de un programa el cual es descrito en la siguiente sección. Cabe mencionar que el experimento es llevado a cabo en un cuarto oscuro, con el fin de evitar la luz parasita.

\section{Imagen sólida}

La imagen sólida de una reacción enzimática, en esta investigación, se refiere a la imagen digital obtenida por un microscopio conectado una cámara digital, en 
este caso no es el interés la estructura presente en la imagen digital, sino el valor promedio de los pixeles en la imagen de luminancia ${ }^{[8]}$, el cual es empleado en el cálculo de la absorbancia. El procedimiento para obtener este valor es el siguiente: Se captura una imagen sólida con una cámara digital CMOS, esta imagen es descompuesta en sus componentes RGB, las cuales son empleadas para obtener una imagen de luminancia relativa de acuerdo a la norma CIE XYZ ${ }^{[9]}$ posteriormente se calcula la intensidad promedio Im de todos sus pixeles, aproximando al valor más próximo. Este valor es empleado para calcular la absorbancia correspondiente a la imagen sólida empleando la Ecuación (1) y considerando Ir=251. Este procedimiento se muestra en la Figura 2.
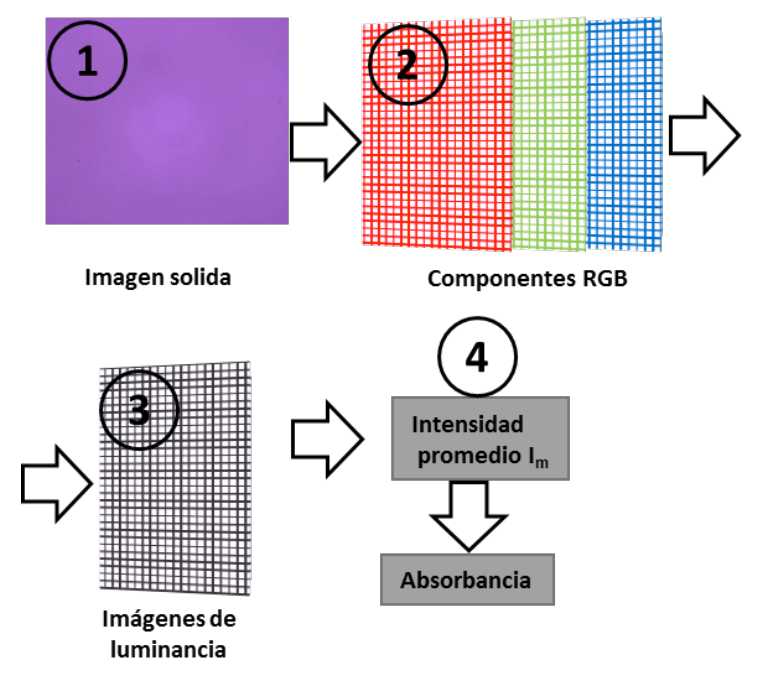

FIGURA 2. Procedimiento de conversión

de imagen sólida a valor de absorbancia.

Un ejemplo de una secuencia de imágenes solidas de violeta de genciana para concentraciones en aumento, son presentadas en la Figura 3, en cada caso se obtienen el promedio de intensidad de los pixeles en la imagen de luminancia, y como se puede observar esta disminuye conforme aumenta la concentración del colorante, mientras que la absorbancia aumenta progresivamente. En estas imágenes solidas no existe alguna estructura, ya que la intensidad promedio y el valor de absorbancia es la de interés.

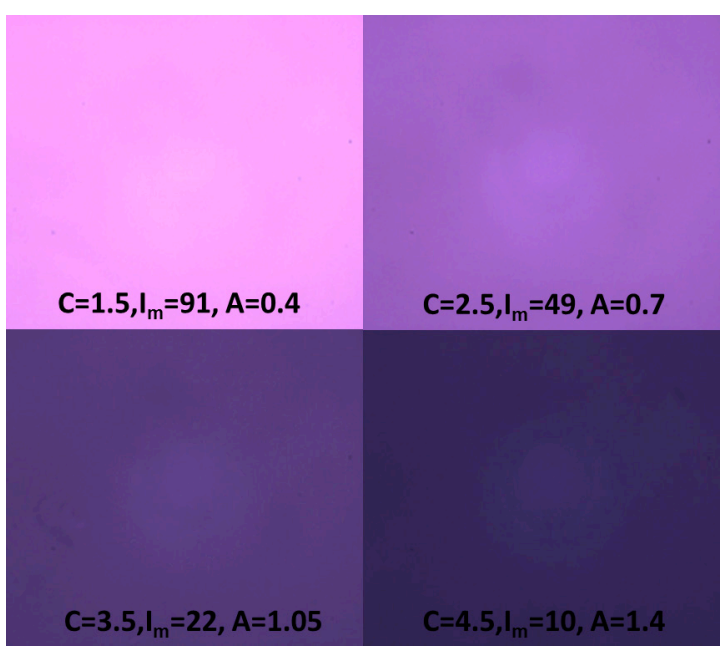

FIGURA 3. Secuencia de imágenes solidas de colorante violeta de genciana para concentraciones de 1.5 a $4.5 \mathrm{mg} / \mathrm{dL}$. Para cada imagen C=concentración $\mathrm{mg} / \mathrm{dL}, \mathrm{Im}=$ Intensidad promedio

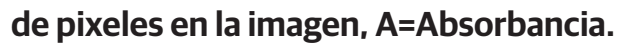

\section{Programa para el cálculo de Absorbancia y concentración}

Se implementó un programa que realiza mediciones de absorbancia y concentración a partir de imágenes solidas de solutos, este programa se realizó en el software Labview 2015 y utilizando el toolkit visión [10], este último permite la activación y captura de la cámara CMOS, además de contar con funciones especializadas en el procesamiento de imágenes.

El programa cuenta dos opciones, medición de absorbancia (A) y medición de concentración (C), que el usuario puede elegir de acuerdo a sus necesidades (ver Figura 4). Para el caso de medición de absorbancia, el programa solicita al usuario introducir el número de lecturas (1) a realizar, posteriormente el usuario ubica el primer poso a leer en la placa de Elisa utilizando la platina x, y (2) del microscopio; a continuación el programa activa la cámara CMOS y captura una imagen (3) de tamaño 1600x1200 pixeles. Las imágenes RGB de la imagen captura son extraídas y empleadas para obtener una imagen de luminancia (4) de la cual se obtiene la intensidad promedio $I_{m}$ (5) de todos sus 
pixeles, redondeado al valor más próximo. Este valor es empleado para calcular la absorbancia correspondiente a la imagen sólida en turno (Ecuación (1) y considerando $\mathrm{Ir}=251$ ). El proceso de conversión de imagen solida a medición de absorbancia, es mostrado en la Figura 2. Posteriormente el programa retorna al paso 2 y el usuario ubica un nuevo poso a leer. Finalmente, las $\mathrm{n}$ mediciones de absorbancia son almacenadas en un vector (6) que contiene las relaciones de concentración conocidas y sus mediciones de absorbancia.

Para la opción de la medición de concentración el vector que contiene las relaciones de concentración y absorbancia es utilizado para obtener una recta de calibración (7) por medio de una regresión lineal, esta recta es del tipo:

$$
\mathrm{A}=\mathrm{mc}+\mathrm{b} \rightarrow \mathrm{c}=\mathrm{A}-\mathrm{b} / \mathrm{m}
$$

Dónde: A es la absorbancia, $\mathrm{m}$ es la pendiente de la recta, c es la concentración, y b es la intersección con el eje de la absorbancia. Por último, el programa estima concentración (8) a partir del despeje de la recta de calibración en términos de la concentración (ver Ecuación (3)). El diagrama de flujo del programa es mostrado en la Figura 4.

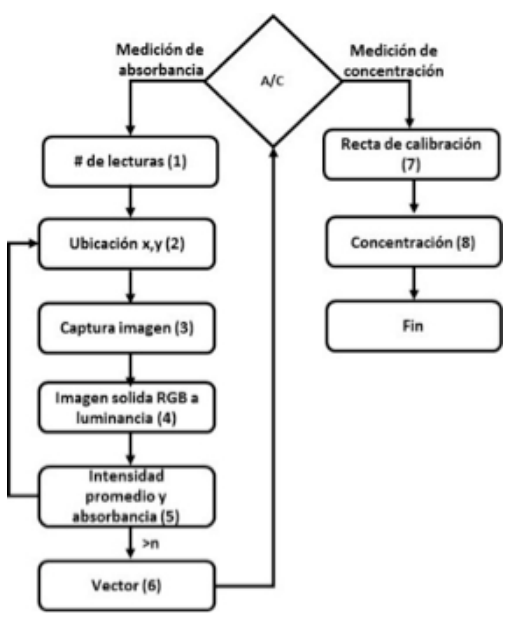

FIGURA 4. Pasos que realiza el programa para obtener mediciones de absorbancia y concentración de reacciones enzimáticas.

\section{RESULTADOS}

\section{Colorante de utilidad clínica}

Con el único fin de analizar el desempeño del MLA, se realizó comparación cuantitativa de mediciones de absorbancia entre un lector de elisa modelo H Reader 1 y el sistema propuesto. Para lo anterior, se emplearon diferentes concentraciones de un colorante de utilidad clínica como lo es el violeta de genciana $\left(\mathrm{C}_{24} \mathrm{H}_{28} \mathrm{~N}_{3} \mathrm{Cl}\right.$ al $1 \%$ en agua destilada), el cual usualmente se emplea para la realización de curvas de calibración en determinación de metabolitos en sangre. Se preparó una solución stock de violeta de genciana a una concentración de $100 \mathrm{mg} / \mathrm{dL}$, y se obtuvieron disoluciones en agua destilada de 1.5 a 6.5 con variación de $1 \mathrm{mg} / \mathrm{dL}$ (C en la columna uno de la Tabla 2). Las mediciones de absorbancia para los dos lectores son mostradas en la siguiente tabla.

TABLA 2. Tabla comparativa de mediciones absorbancia y concentración en ELISA y MLA, para diferentes concentraciones de violeta de genciana.

\begin{tabular}{|ccccl|}
\hline C & Abs-E & Abs-MLA & C-MLA & E-C \\
\hline $\mathbf{1 . 5}$ & 0.439 & $0.441-(91)$ & 1.506 & 0.006 \\
\hline $\mathbf{2 . 5}$ & 0.708 & $0.709-(49)$ & 2.383 & 0.117 \\
\hline $\mathbf{3 . 5}$ & 1.06 & $1.057-(22)$ & 3.518 & 0.018 \\
\hline $\mathbf{4 . 5}$ & 1.371 & $1.400-(10)$ & 4.636 & 0.136 \\
\hline $\mathbf{5 . 5}$ & 1.682 & $1.701-(5)$ & 5.618 & 0.118 \\
\hline $\mathbf{6 . 5}$ & 1.972 & $1.923-(3)$ & 6.342 & 0.158 \\
\hline
\end{tabular}

Se puede observar en la Tabla 2 que las mediciones de abs-E y abs-MLA (mediciones de absorbancia del lector de ELISA y el sistema propuesto respectivamente) son muy cercanas unas a otras, por lo tanto, podemos intuir que el sistema MLA es comparable con lectores de absorbancia comerciales.

Por otro lado, con el fin de cuantificar la exactitud en la medición de concentraciones por parte del MLA, se obtuvo la recta de calibración del sistema aplicando 
una regresión lineal a los datos de concentración y Abs-MLA obteniendo un R2 $=0.9959$. Dicha ecuación lineal es despejada con respecto a la concentración para su correspondiente cálculo, como se muestra en la siguiente ecuación:

$$
\mathrm{C}-\mathrm{MLA}=\mathrm{A}+0.0207 / 0.3064
$$

Dónde: C-MLA es la concentración estimada en mg/ dL, A es la absorbancia, 0.0207 es la intersección en el eje de la absorbancia y 0.3064 pendiente de la recta de calibración. Así mismo el error en la medición en la concentración (E-C) es mostrado en la cuarta columna de la tabla dos, el cual es calculado a partir del valor absoluto de la diferencia de la concentración experimental con la concentración calculada con la ecuación cuatro, en esta columna se observa que el error aumenta conforme aumenta la concentración, esto puede deberse a que la diferencia entre intensidad de niveles de gris de imágenes digitales de soluciones, a concentraciones altas es muy pequeña. Por ejemplo, para concentraciones de 5.5 y $6.5 \mathrm{mg} / \mathrm{dL}$ la variación de intensidad es tan solo de dos en una escala de grises. Lo anterior también se puede verificar observando la Figura 5, ya que ambas graficas se separan más a concentraciones altas.

\section{Recta de calibración de glucosa}

Fue realizada una recta de calibración con el reactivo líquido para la determinación fotométrica de glucosa en suero o plasma del Grupo Mexlab ${ }^{[11]}$, con el objetivo de cuantificar el desempeño del MLA comparado con un lector de ELISA empleado en la investigación. La recta de calibración del sistema aplicando una regresión lineal a los datos de concentración y Abs-MLA fue obtenida con un R2 =0.9991. Dicha recta de calibración es despejada con respecto a la concentración para su cálculo, como se muestra en la siguiente ecuación:

$$
\mathrm{C}=\mathrm{A}-0.0166 / 0.0085
$$

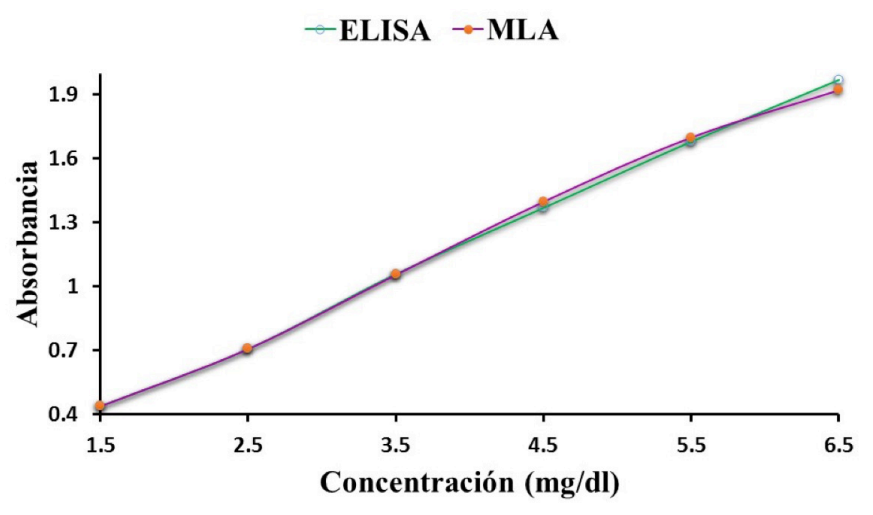

FIGURA 5. Grafica comparativa de mediciones de absorbancia de abs-E y abs-MLA.

Dónde: C-MLA es la concentración estimada por el sistema propuesto en $\mathrm{mg} / \mathrm{dL}$, A es la absorbancia, 0.0207 es la intersección en el eje de la absorbancia y 0.3064 pendiente de la recta de calibración. En la tabla tres se muestran las mediciones de absorbancia de ambos lectores (Abs-E y Abs-MLA), columna dos y tres, mientras que en la cuarta y quinta columna son mostrados las concentraciones estimadas con la Ecuación cinco (C-MLA) y su error respectivamente (E-C).

\section{TABLA 3. Mediciones absorbancia y concentración en elisa y MLA, para diferentes concentraciones del reactivo líquido para la determinación fotométrica de glucosa en suero.}

\begin{tabular}{|ccccc|}
\hline $\mathrm{C}$ & Abs-E & Abs-MLA & C-MLA & E-C \\
\hline $\mathbf{7 5}$ & 0.669 & $0.651-(56)$ & 74.635 & 0.365 \\
\hline $\mathbf{1 0 0}$ & 0.894 & $0.868-(34)$ & 100.164 & 0.164 \\
\hline $\mathbf{1 2 5}$ & 1.119 & $1.077-(21)$ & 124.752 & 0.248 \\
\hline $\mathbf{1 5 0}$ & 1.344 & $1.286-(13)$ & 149.341 & 0.659 \\
\hline $\mathbf{1 7 5}$ & 1.569 & $1.497-(8)$ & 174.164 & 0.836 \\
\hline $\mathbf{2 0 0}$ & 1.794 & $1.701-(5)$ & 198.164 & 1.836 \\
\hline
\end{tabular}

La comparación de las rectas de calibración para los dos sistemas se muestra en la Figura 6, donde se puede notar que a concentraciones altas ambas se empiezan a separar, posiblemente porque la cámara digital CMOS tiene menos sensibilidad a concentraciones altas. 


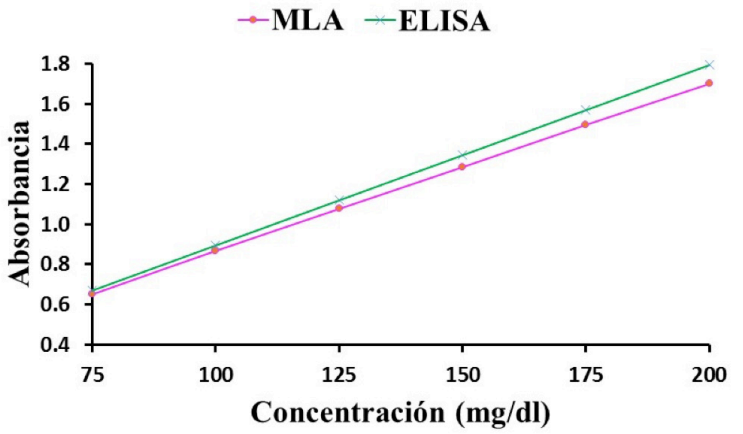

FIGURA 6. Gráfica comparativa de mediciones

de absorbancia de Abs-E y Abs-MLA, para el caso

del reactivo líquido para la determinación

fotométrica de glucosa en suero.

\section{Utilidad clínica del MLA}

Uno de los objetivos principales de esta investigación, es el empleo del MLA como una alternativa a lectores de absorbancia convencionales, para análisis clínicos, en este caso el sistema tiene una doble funcionalidad, ya que puede servir como microscopio o cuantificador de concentraciones.

Por lo anterior se realizó la determinación de glucosa en muestras problemas de pacientes voluntarios de la Universidad de la Cañada, otorgándose al individuo una copia del consentimiento informado para el aseguramiento de su participación en esta investigación.

Se formaron dos grupos de tres pacientes, el primer grupo son individuos clínicamente sanos y el otro grupo fueron diagnosticados previamente como hipoglucémicos. A estos grupos se les extrajo $5 \mathrm{~mL}$ de plasma sanguíneo sin coagulante, después de dejar coagular la muestra, se continuo a centrifugar las muestras a 3500 rpm durante 5 minutos, para la obtención de suero sanguíneo. Una vez obtenidas la muestras, se realizó la reacción enzimática de Trinder GodPod ${ }^{[11]}$ y finalmente las mediciones de concentraciones fueron estimadas empleando el analizador de química sanguínea semiautomatizado (AQSA), modelo Easykem plus y el MLA, para este último caso, la concentración fue estimada a partir de la ecuación cinco correspondiente a la recta de calibración glucosa. Los resultados de ambos equipos son comparados en la Tabla 4 .

\section{TABLA 4. Tabla comparativa de mediciones absorbancia} y concentración en AQSA y MLA, para seis pacientes.

\begin{tabular}{|ccccc|}
\hline PC & C-AQSA & Abs-MLA & C-MLA & E-C \\
\hline $\mathbf{1}$ & 398.56 & ---- & --- & --- \\
\hline $\mathbf{2}$ & 282.199 & ---- & --- & ---- \\
\hline $\mathbf{3}$ & 145.812 & $1.254(14)$ & 145.576 & 0.236 \\
\hline $\mathbf{4}$ & 92.408 & $0.798(40)$ & 91.929 & 0.479 \\
\hline $\mathbf{5}$ & 73.953 & $0.651(56)$ & 74.635 & 0.682 \\
\hline $\mathbf{6}$ & 62.435 & $0.548(71)$ & 62.517 & 0.082 \\
\hline
\end{tabular}

PC: Número de paciente. C-AQSA: Medición de concentración con AQSA en $\mathrm{mg} / \mathrm{dL}$. Abs-MLA y C-MLA: Lecturas de absorbancia y concentración del sistema propuesto respectivamente. E-C: Error en concentración, calculado a partir de C-AQSA menos C-MLA.

Como puede observarse en la tabla cuatro, los pacientes uno y dos presentaron rangos de medición de absorbancia fuera del rango, permitido por el MLA, mientras que los otros cuatro pacientes están dentro del rango de medición, también puede observarse que los errores de medición no fueron mayores a $1 \mathrm{mg} / \mathrm{dL}$.

\section{DISCUSIÓN}

De acuerdo a las mediciones obtenidas con lectores de absorbancia comerciales, estas son semejantes a las mediciones obtenidas con el MLA, arrojando un error no mayor a $1 \mathrm{mg} / \mathrm{dL}$ para los casos de los resultados del colorante, glucosa y muestras problemas de pacientes voluntarios (ver Tabla 2, Tabla 3, y Tabla 4). Para estos casos las rectas de calibración presentaron un R2 alto y una pendiente baja, lo cual indica que el sistema propuesto presenta una buena exactitud y precisión en mediciones arbitrarias (ver Figura 5 y Figura 6). Sin embargo, el límite de detección está acotado, debido a la sensibilidad de la CMOS que se empleó, ya que, a concentraciones altas, la intensidad de luz que llega al detector es muy poca y esta puede ser confundida con el ruido térmico generado por la CMOS, (ver Tabla 2). 
Por otro lado, para intensidades altas (concentraciones bajas) la sensibilidad del sistema se reduce ya que los cambios de nivel de gris en la imagen solida son insignificantes (ver Figura 6). Sin embargo, empleando un rango de medición de absorbancia de 0.4 a 0.9, el sistema propuesto presenta una alta linealidad. Si se desea aumentar el rango de medición, el sistema debe de sufrir dos modificaciones, la primera consistiría en tener una cámara digital con un ruido térmico más bajo y la segunda consistiría en aumentar la intensidad de la fuente de iluminación, en ambos casos, una recta de calibración debe ser obtenida para una correcta medición en el MLA.

Por otro lado, la conjunción de la recta de calibración con el algoritmo, es de suma importancia, ya que este último calcula las concentraciones a partir de la recta de calibración es decir a partir de la pendiente y la intersección en el eje de absorbancia, por lo que un cálculo no adecuado de estos, arrojaría resultados erróneos (ver Ecuaciones 3, 4 y 5). Teniendo en cuenta lo anterior el sistema resulta ser una alternativa viable para la estimación de absorbancia, y proporciona a los microscopios ópticos una doble funcionalidad.

\section{CONCLUSIONES}

La adaptación de un filtro de luz, una CCD y un algoritmo computacional, a un microscopio óptico, aumentan sus cualidades como instrumento de medición, en este caso como medidor de absorbancia, para estimar concentraciones desconocidas. Lo anterior brinda la posibilidad de realizar mediciones cerca del punto de atención del paciente, ya que los microscopios ópticos son de uso común en clínicas de salud. A su vez el sistema brinda la posibilidad de la reducción de reactivos al poder calcular absorbancias de áreas microscópicas, por otro lado, en trabajos futuros, esta propuesta se pretende seguir validando, de acuerdo a normas establecidas, patrones de referencia y múltiples ensayos, con el objetivo de determinar los criterios de validez del método, así como la exactitud del método, precisión, linealidad, entre otros. 


\section{REFERENCIAS}

[1] Grahn HF, Geladi, P. Technique and Applications of Hyperspectral Image Analysis. The Atrium, Southern Gate, Chichester, West Sussex, PO19 8SQ, England: John Wiley \& Sons, 2007.

[2] Christopher P Price. Regular review Point of care testing. bmj volume 32226 may 2001.

[3] Greenfield Sluder and David E. Wolf. Digital Microscopy 3RD edition, Volume 81, Pages 64-67 (2007).

[4] Peter D. Hiscocks, P.Eng. Measuring Luminance with a Digital Camera, Syscomp Electronic Design Limited, February 16, 2014.

[5] Olympus, inverted microscope CKX41, 2016.

[6] Thorlabs. (2003). FB510-10. agosto 7,2018, de Thorlabs Sitio web: https://www.thorlabs.com/thorproduct.cfm?partnumber $=$ FB510-10

[7] Lumenera Corporation. (2017). INFINITY1-2. julio,2018, de Lumenera Corporation Sitio web: https://www.lumenera.com/ infinity1-2.html
[8] Anastas Dakashev,Stanch Pavlov,Krasimira Stancheva, Application of Digital Camera and Digital Image Processing Techmique for Molecular Absorption Analysis in the Visible Spectrum, Universal Journal of Chemistry Vol. 1(4), pp. 129 - 134 DOI: 10.13189/ujc.2013.010401

[9] Carranza-Gallardo, J. "Manejo de las fórmulas de diferencias de color vs límites de aceptabilidad". Memorias del Simposio de metrología 2002, Centro Nacional de Metrología, México., 2002.

[10] National Instruments. (2005). NI Vision. julio,2018, de National Instruments Sitio web: $h t t p: / / w w w . n i . c o m / p d f / m a n u a l s / 371007 b$. $p d f$

[11] Grupo Mexlab. Bio-Glucosa, Reactivo para la determinación fotométrica de glucosa en suero o palsma y otros fluidos biológicos. Sitio web: http://www.grupomexlab.com 